\title{
Relative Planar Strain Control and Minimizing Deformation Work in Elastomeric Sheets via Reinforcing Fiber Arrays
}

\author{
Michael Kriega,c, Kamran Mohsenia ${ }^{\mathrm{a}, \mathrm{b}, \mathrm{c}, *}$ \\ University of Florida, Gainesville, FL \\ ${ }^{a}$ Department of Mechanical and Aerospace Engineering \\ ${ }^{b}$ Department of Electrical and Computer Engineering \\ ${ }^{c}$ Institute for Networked Autonomous Systems
}

\begin{abstract}
This study investigates soft composite sheets that undergo significant deformations. The fiber reinforcement in these systems not only increases the stiffness of sheets like a traditional composite, but also controls the relationships between strains in orthogonal planar directions. Such an ability is useful in controlling the deformation of soft robots, and also enhancing the output of soft actuation techniques like electro-active polymer actuators (also called dielectric actuators). The inspiration for this work comes from squid mantle structures that couple orthogonal components of strain using helical fiber reinforcement,. The resulting null space of deformations corresponding to the fiber restrictions creates a family of body deformations that optimize propulsion.

The strain dynamics in the composite sheet are modeled geometrically from fiber orientations, assuming that the fibers are inextensible. After the strain dynamics have been determined, the stress/strain relationship is modeled by considering the matrix and reinforcing fibers to be two separate homogeneous systems interacting through local stresses. Both steps of this modeling technique are validated experimentally showing planar strains in a preferred direction to be as high as 16 times the resulting planar strain of an equivalent unreinforced sheet by forcing negative strains in the orthogonal planar directions. The work required for deformation is derived from the stress-strain relationship by calculating the strain energy stored in the material, and an optimal balance between increased planar strain output and increased material stiffness is analyzed. It is shown that for the specific materials used to create the soft composite sheets (thermoplastic elastomer with cotton fibers) optimal fiber angles lie between $15^{\circ}$ and $25^{\circ}$ to minimize work required for deformation, but this optimal range will increase with increasing ratio of fiber to matrix modulus.
\end{abstract}

Keywords: Flexible Composites, Deformation, Non-linear Behavior, Stress Transfer

2010 MSC: 00-01, 99-00

\section{Introduction}

Much of the inspiration for this investigation into soft composite sheets comes from observations that fiber reinforcement in soft biological tissues serves a skeletal role, as well as increasing material stiffness [1]. Flexibility is a defining property of propulsion in marine animals [2]. In fish and marine mammals, body shape is dictated by an internal rigid skeleton, and flexible tissues are stretched over the internal structures. However, there are appendages

\footnotetext{
* Corresponding author

Email address: support@elsevier.com (Kamran Mohseni)
}

Preprint submitted to Composites: Science and Technology

December 13, 2016

(C) 2017. This manuscript version is made available under the Elsevier user license http://www.elsevier.com/open-access/userlicense/1.0/ 

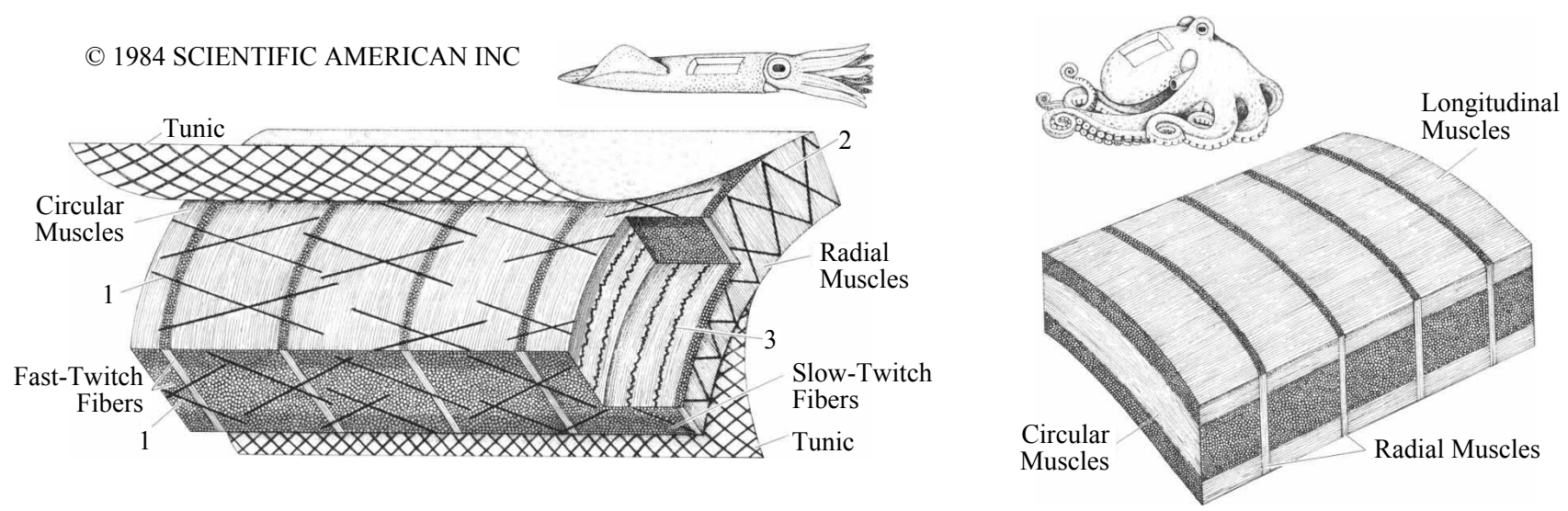

Figure 1: (left) Schematic of squid mantle tissue dominated by circular muscle fibers with both inner and outer tunics. (right) Schematic of octopus mantle tissue with circular, radial, and longitudinal muscle tissue. Figures taken from 5 ]

and entire animals that are able to maintain their basic shape without any such rigid elements. Often they rely on systems known as 'muscular hydrostats' [3]. In structures such as these rigid skeletal elements are completely absent, instead arrangements of muscles and fibers provide both the forces to drive motion, as well as the support to maintain the desired system geometry. Such systems include elephant trunks and the tongues of various mammals 4 to name 10 a few.

When it comes to soft bodies with no rigid (skeletal) elements whatsoever, there is a trade-off that exists between the possible versatility of the body's movements and high performance of a specialized action. This trade-off exists in biological organisms as well as engineered systems. Here, we summarize, as a prime example, the differences in the mantle structures of squid and octopuses. The mantle is a hollow muscular cylinder surrounding the visceral mass of cephalopods (e.g. squid, octopus, cuttlefish) that is periodically filled with water for respiration and jet propulsion. Figure 1. which is composed of two figures from [5], illustrates the structure of a portion of each animal's mantle wall. The octopus mantle tissue is composed of three orthogonal muscle groups, i.e. circumferential, radial, and longitudinal, and by activating these muscle groups in various combinations can deform its body to nearly any shape. This versatility is evidenced by the abundance of complex behaviors exhibited by various octopus, from camouflaging themselves with a combination of changing body shape and using a complex system of chromatophores, to their ability to pass through tiny openings much smaller than their overall body size.

The squid mantle, on the other hand, is specialized to provide the most effective jetting of any cephalopod. Squid mantles are encompassed by an inner and outer shell of interwoven collagen fibers called tunics (see figure 1). The tunic fiber angle is surprisingly uniform from squid to squid. Ward \& Wainwright [6] observed tunic fiber angles of several specimen of Lolliguncula brevis to be at $27^{\circ} \pm 1^{\circ}$. If the mantle is assumed to be cylindrical and the fibers to be inextensible, then there is a unique cylinder length and radius for every tunic fiber angle. Vogel, following the example of other studies on helically wound biological reinforcing fibers [7], plotted the volume of the mantle cavity as a function of the fiber angle, showing that for acute tunic fiber angles, a decrease in mantle circumference results in a slight elongation, but a net decrease in mantle volume corresponding to jet ejection [8]. Krieg \& Mohseni [1], having 30 noticed that squid only eject a fraction of the total mantle volume during jetting, demonstrated that the rate of change of a cylindrical mantle volume with respect to diameter contraction reaches a maximum when the fiber angle is at 
$\approx 31^{\circ}$, very close to the actual tunic fiber angles observed in [6]. This means that the tunic fiber angles are aligned to provide maximum propulsive jet volume, for a given circumferential muscle contraction. It can be understood in this context that the role of the tunic fibers in the squid mantle is to control the relationship between the circumferential and longitudinal components of strain in the flexible mantle. During jetting the mantle tissue becomes thicker with the decreasing diameter because the tissue resists volume change, and the tissue extends in the axial direction, the relationship between the axial and azimuthal strain being controlled by the tunic fiber angle.

Since the deformation of the squid mantle in the axial direction is limited by the tunic fibers, it does not require longitudinal muscle groups to oppose this extension. As a result, more of the muscle in the mantle can be dedicated In addition, using tunic fibers to restrict deformation of the squid in the longitudinal direction is a passive process, whereas using longitudinal muscle groups to limit extension requires appreciable energetic input. So the tunic fibers also increase the efficiency of the deformation process. Squid mantles also contain an array of flexible intramuscular (IM) fibers that stretch as the mantle wall thickens during jetting, storing elastic potential energy that re-expands the 45 mangle following jetting and thereby aids refilling the mantle cavity [10, 11, 12, 13. The action of the IM fibers, reduces the need for radial muscle groups during refilling, and allows for a further increase in the amount of circular muscle groups driving jetting. It was shown specifically that IM-3 fibers' orientation allows for $90 \%$ of the maximum possible potential energy storage [1]. The specialized mantle structure gives squid impressive locomotory capabilities, including the fastest swimming speeds of any marine invertebrate [14, 15. For a more complete summary of reinforcing fiber arrays in biological systems, please refer to [7. In particular, the discussion about how the fiber angle of reinforcing helical fibers in the cuticles of nematodes and other worm-like organisms controls the stretching of the worm length relative to the contraction of worm width is a concept which is investigated at length in this study.

In a mathematical sense, the trade-off between versatility and specialized performance can be described as a restriction of the deformation space of a soft body. To help explain this concept, consider a system of rigid elements connected by a series of joints. The exact configuration of the system can be uniquely defined by the angle at each connecting joint, meaning that the deformation space of the system has a finite number of degrees of freedom equal to the number of joints. Conversely, a completely deformable body, with no rigid elements, has an infinite number of degrees of freedom. By embedding the soft body with inextensible reinforcing fibers, the original infinite deformation space is reduced to a null space of possible geometries that satisfy the lack of extension in the fibers. The advantages ${ }_{60}$ and disadvantages of this process are, as just mentioned with the cephalopod mantle example, that muscles/actuators can be increased in a desired direction, since they are not required to counter deformation in the directions limited by the fibers, but the resulting range of movement is restricted.

The role of reinforcing fibers can go beyond just restricting unwanted deformation, it can in fact drive negative strains in unwanted directions to further increase extension in a desired direction. As an example, one common actuation technique used in the field of soft robotics is Electro-Active Polymer actuators (EAPs), sometimes called dielectric elastomer actuators (DEAs), due to their fast response times, high power density, and large degree of flexibility [16. The basic concept of this type of actuator is that flexible conducting plates sandwiching a layer of elastomer are charged, squeezing the plates together due to capacitive force, resulting in an outward expansion of the elastomer layer by conservation of volume. It has been shown that the performance of EAPs driving motion in a single 
Section of Elastomer

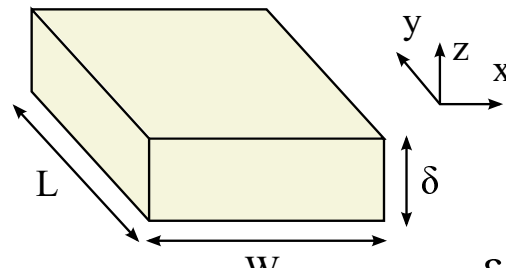

Principle Axis Strains $\varepsilon_{\mathrm{x}}=\frac{\Delta \mathrm{W}}{\mathrm{W}} \quad \varepsilon_{\mathrm{y}}=\frac{\Delta \mathrm{L}}{\mathrm{L}}$

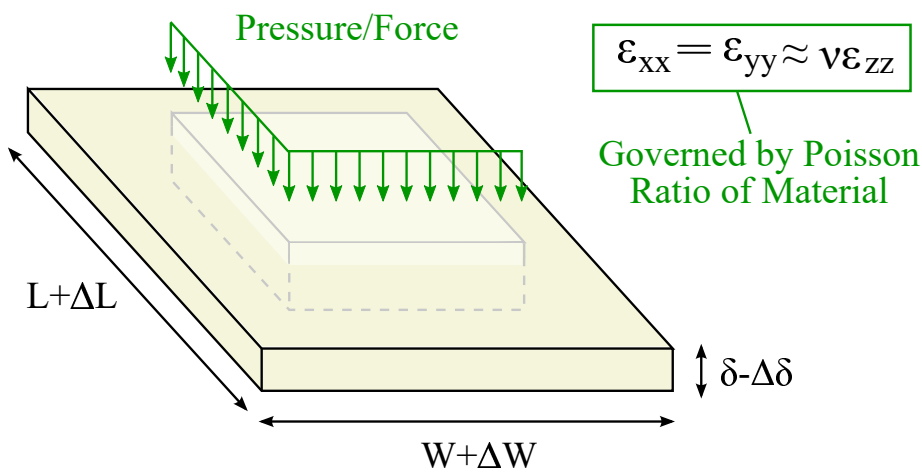

Fiber Angle

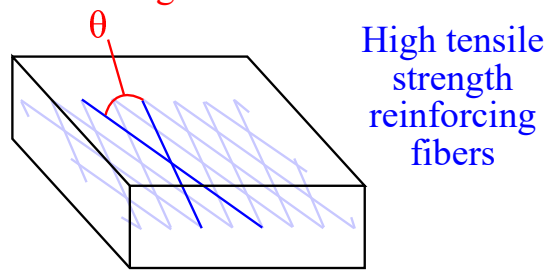

Increased Fiber Angle Constant Fiber Length

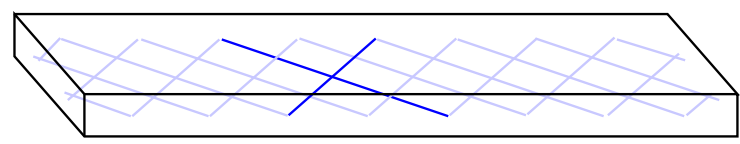

Relative Strain Controlled by Fiber Angle

Figure 2: Diagram illustrating the basic mechanics of a soft composite with tunable strain properties. of prestraining EAPs is mostly due to the fact that prestraining increases the dielectric constant of the elastomer, but also partially lies in the ability to control the relative strains in each individual direction by changing the stress-strain properties. When the elastomer is compressed, more force is required to stretch in the planar direction that is already prestrained. As a result, there is an increased strain in the desired planar direction. An alternative but unexplored relative amounts of strain in each direction, as described above for the mantle tunic fibers. The fundamentals of this method are as follows.

Figure 2 shows a schematic diagram of the basic concept. On the left is shown a basic section of elastomer. Under compressive forcing, the elastomer expands outwards in the $x$ and $y$ directions. The sum of strains in the $x$ and $y$ directions is governed by the Poisson ratio of the elastomer, but the strains in each direction relative to each other are governed by the geometry and homogeneity of the elastomer. If fibers with high tensile stiffness are suspended within the elastomer, then the material is free to bend and stretch in directions tangent to the fibers, but is restricted in the direction along the fiber's axis. Therefore, if all the fibers are aligned parallel in a given direction then the resulting planar strain after compression will only exist perpendicular to the fibers. If alternatively, the fibers are aligned at angle, which under constant fiber length assumptions actually results in a negative strain in the opposite direction. Therefore, by embedding reinforcing fibers, the soft material can be made to deform only in a single direction or even increase desired strain by driving negative strain in the orthogonal planar direction. Adjusting the fiber's tensile stiffness allows the relative strains in the lateral direction to be controlled to any desired value. The current study,

The use of reinforcing fibers in elastomer composites has become commonplace in bicycle tire fabrication [18. 
Hence, there has been some attempt to ascertain the anisotropic stress-strain properties of these materials. The fabrication process for this type of application, however, generally introduces fibers into the elastomer matrix as a pulp containing discontinuous short fibers that, even after processing, only attain a statistical distribution around any given axial orientation. Furthermore, tire and belting applications are mostly concerned with increasing the stiffness and tear resistance of stiffer classes of rubbers, so experimental analysis has largely focused on statistical increases in stiffness, rather than the alteration of stress dynamics within the soft composite material. This modeling has been very similar to traditional laminate theory used to model composites. Accordingly, composites are assumed to be homogeneous at large scales, but anisotropic, and the modulus of the composite material is calculated from the individual moduli of the matrix and the fibers and their respective volume fractions, along with an efficiency or Krenchel factor accounting for the fiber reinforcement [19].

The few studies that take a more in depth look at the material deformation dynamics in fiber reinforced elastomers have typically taken this traditional approach. Experimental studies have characterized the non-linear elastic modulus of soft composites for a variety of fiber volume fractions, for straight [18] and wavy or zig-zag [20, 21] reinforcing fibers. A large degree of stiffening has been observed, associated with fiber straightening and reorientation, but observed nonlinearity is typically fitted to heuristically determined curves. Clark [20] observed a bi-linear stress/strain dynamic for the zig-zag fibers, and Lou and Chou [21] fit the strain energy to a 4'th order polynomial function of the strain for both straight and wavy fibers. Peel performed tensile testing of soft composite struts and modeled the stress/strain relationship with a non-linear laminate model [22], though again the nonlinear behavior is attributed to fiber reorientation, but not directly modeled. Helical fiber orientation has also been identified as a parameter that directly affects the performance of pneumatic actuators [23. However, similar to the studies just mentioned, the output is directly correlated to actuator pressure without modeling the strain dynamics in the pneumatic tube.

Our approach assumes inextensible fibers to first solve for the strain dynamics of the soft composite sheet. Next, the stress/strain relationship is modeled by considering the elastomer and fiber arrays to be independent systems that interact through local planar stresses required to generate the strains determined geometrically. Finally the strain energy stored in the composite is calculated for the elastomer and fibers separately, having relaxed the inextensible fiber assumption.

In section 2 derives the strain dynamics of the sheets assuming that the length of the reinforcing fibers remains constant. The stress interactions between the fibers and matrix under the solved strain dynamics are the presented in section 3 . The experimental apparatus used to validate the modeling is described in section 4 and the resulting performance, model accuracy, and model shortcomings are provided in section 5 In addition, section 5 predicts optimal fiber angles that minimize potential energy storage in the material under various conditions.

\section{Modeling Planar Strain of Soft Composites}

This first section of the soft composite sheet model derives relationships between the primary components of strain force interactions between the elastomer and the fibers based on the kinematic relationships defined here.

Consider a flexible sheet with an array of regularly spaced fibers embedded within it, as illustrated in figure 3 . For the purposes of this model we will assume that the fibers are inextensible, but provide no resistance to bending, and 


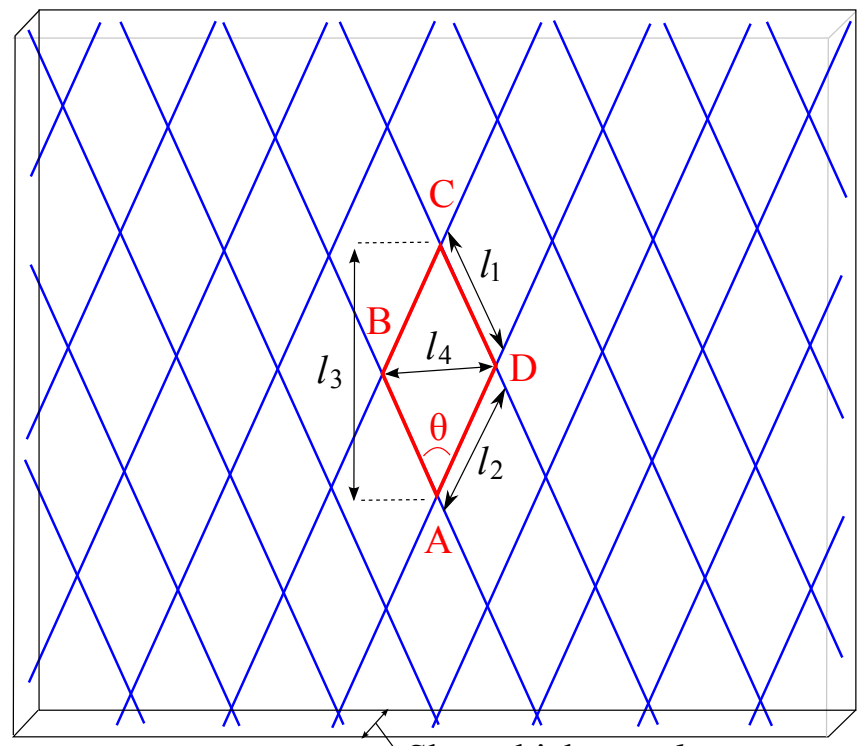

Sheet thickness, $h$

\section{Characteristic Directions and Angles for Unequal Fiber Lengths}

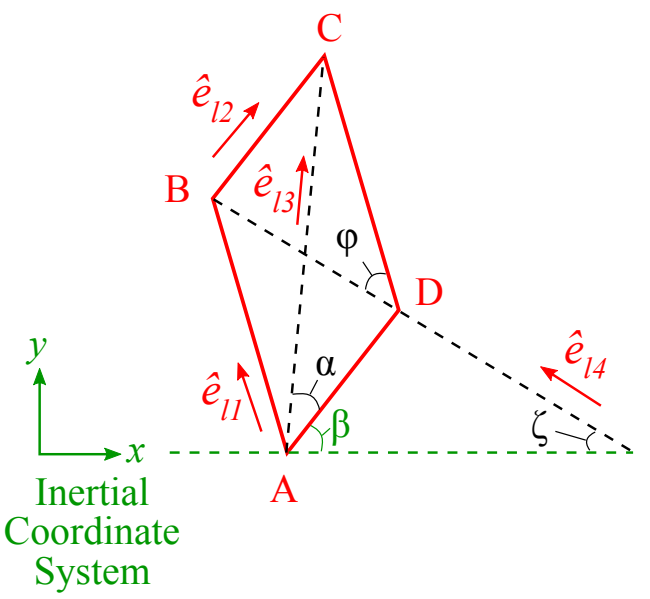

Figure 3: Illustration showing a network of fibers suspended in an elastic matrix sheet, highlighting the smallest basic element of the array.

that there is perfect bonding between the fibers and the surrounding elastomer matrix. For this primary investigation, however, we assume that stress loading is uniform, so that bending will not be considered in the fibers, despite the lack of bending stiffness.

\subsection{Conservation of Volume and Poisson's Ratio}

Poisson's ratio, $\nu$, which is the opposite of the ratio of strain in the transverse directions to the strain in a direction under loading, is typically characterized for different materials and treated as a constant for small deformations. This ratio is directly coupled to the compressibility of a material. If a material is compressed in one direction, and it is incompressible, then it must expand out in the transverse directions. For very soft materials, like the elastomer used as a matrix in this study, the low resistance to deformation results in volume being very nearly conserved in most deformations. Stiffer materials, on the other hand, experience more compression and have lower Poisson ratios.

For small material strains, conservation of volume is equivalent to a Poisson ratio of $\nu=0.5$. This is not the case for larger deformations. Consider a cube that is placed under compressive strain, which we will refer to as the $z$-direction. As the cube is compressed down to an infinitesimal thickness, the length scales in the $x$ and $y$ directions grow to infinity. Since the $z$ strain ranges from 0 to -1 , while the planar strains range from 0 to $\infty$, the Poisson ratio ranges from 0.5 to $\infty$ depending on the scale of the deformation.

The compressibility of a material can also be characterized by a term called the volumetric strain or compressibility strain, which is defined,

$$
\epsilon_{V}=\frac{V-V_{0}}{V_{0}}=\left(1+\epsilon_{x x}\right)\left(1+\epsilon_{y y}\right)\left(1+\epsilon_{z z}\right)-1
$$

where $V$ is the volume of a material element, $\epsilon_{x x}, \epsilon_{y y}$, and $\epsilon_{z z}$ are the strains of the material element in the Cartesian directions, and the subscript 0 refers to the value taken at an initial undeformed configuration. The kinematic relationships derived here can be described more simply in terms of the volumetric strain, $\epsilon_{V}$, but in all locations the 
volumetric strain can be replaced by the Poisson ratio according to the relationship,

$$
\nu=\frac{1-\left[\frac{1+\epsilon_{V}}{1+\epsilon_{z z}}\right]^{1 / 2}}{\epsilon_{z z}} .
$$

In fact the link between volumetric strain and Poisson ratio has been observed by others, and the relationship between $\epsilon_{V}$ and hydrostatic stress was used to characterize a negative Poisson ratio and negative compressibility in anisotropic foams 24]. For small deformations where volume is conserved $\epsilon_{V}=0$ and $\nu=0.5$, but in general both will be considered variable functions of the loading.

\subsection{Geometric Constraints}

The smallest indivisible element of this soft composite structure is a diamond element in between fiber crossings like diamond ABCD highlighted in figure 3 . Since reinforcing fibers are much stiffer than the elastomeric matrix, they will be considered inextensible in the model. As such, characteristic fiber lengths $l_{1}$ and $l_{2}$ of the basic element will remain constant throughout deformation. Even though figure 3 is drawn with equivalent distances between fibers in different directions, resulting in equivalent lengths $l_{1}$ and $l_{2}$, the theory derived here will be applicable to any configuration of fiber lengths and spacing. In order to maintain a single fiber length scale we introduce the fiber length ratio $\lambda=l_{2} / l_{1}$. We will characterize the orientation of the basic element by the relative angle between fibers, $\theta$. Here it should be noted for the sake of completeness that in biological fiber reinforcement studies fiber angle is typically defined as the angle between fibers and the longitudinal axis of the body, which corresponds to one half of the fiber angle as it is defined here. The fiber angle is defined on the range $\theta \in[0: \pi / 2]$, so that it always refers to the smaller angle in the parallelogram element.

We assume that loading on the sheet is uniform so that the fiber angle, $\theta$, may change, but the fibers remain straight throughout their length. This will not be the case for localized stresses. In addition to uniform compressive stress, it is assumed that the compressive strain is also uniform across the thickness of the sheet.

The volume of the basic element cut out by diamond $\mathrm{ABCD}$ is $\mathrm{Vol}=l_{1}^{2} \lambda \sin (\theta) h$, where $h$ is the thickness of the sheet. When the sheet thickness is reduced the planar area must increase to some degree, depending on how much the material volume is compressed. By assuming that the volumetric strain is some arbitrary function of the sheet parameters, $\epsilon_{V}=f(\theta, h)$, we derive a relationship between the differential change in fiber angle, $d \theta$, for a corresponding differential change in sheet thickness, $d h$,

$$
\sin (\theta) d h+\cos (\theta) h d \theta=\sin (\theta) h\left(\frac{1}{1+\epsilon_{V}}\right)\left(\frac{\partial \epsilon_{V}}{\partial \theta} d \theta+\frac{\partial \epsilon_{V}}{\partial h} d h\right) .
$$

Separation of variables can be used to solve this expression explicitly for the total change in fiber angle to account for a given compressive strain, $\epsilon_{z}$,

$$
\theta_{f}=\arcsin \left(\sin \left(\theta_{0}\right) \frac{1+\epsilon_{V}}{1+\epsilon_{z z}}\right)
$$

It should be noted that there is a maximum fiber angle that can be reached through compression of the soft composite. When the fiber angle reaches $\theta=\pi / 2$ the element attains a maximum area, meaning that any further decrease in sheet thickness can only be balanced by expansion of the elastomer just above or below the fibers themselves, contradicting the assumption that strains are uniform across the thickness, or through significant material compressions. For any given initial fiber angle, the maximum compressive strain is,

$$
\epsilon_{z z, \max }=\sin \left(\theta_{0}\right)\left(1+\epsilon_{V}\left(\theta_{0}, \epsilon_{z z, \max }\right)\right)-1
$$


which requires knowledge of the functionality of the volumetric strain, $\epsilon_{V}$.

\subsection{Planar Strain Components}

Since the fibers are inextensible, and the area of each element can only be altered by changing the fiber angle, the local material does not stretch in the directions normal to the fibers during a given instant of the deformation. We denote the directions of each fiber with the unit vectors $\hat{e}_{l 1}$ and $\hat{e}_{l 2}$, respectively (see figure 3), which change dynamically throughout deformation. The distance between opposing points $\mathrm{AC}$ and $\mathrm{BD}$, denoted by $l_{3}$ and $l_{4}$ respectively, either increase or decrease with deformation depending on whether the compressive strain is positive or negative. The unit vectors in the direction of these lengths are similarly denoted $\hat{e}_{l 3}$ and $\hat{e}_{l 4}$, respectively, and are not necessarily orthogonal if the fiber ratio $\lambda$ is not equal to one.

For an inertial Cartesian coordinate system whose $z$-axis is aligned normal to the composite sheet, the planar strains can be completely described by the orthogonal normal strains $\epsilon_{x x}$ and $\epsilon_{y y}$ and the shear strain $\gamma_{x y}$. The deformation of the element, though, occurs in the characteristic directions $\hat{e}_{l_{3}}$ and $\hat{e}_{l_{4}}$. Since the exact layout of the fiber elements is uniquely determined by the fiber angle, $\theta$, and the fiber scaling, $l_{1}$ and $\lambda$, we define the characteristic deformations with respect to the only parameter not held constant,

$$
\begin{aligned}
& \frac{d l_{3}}{d \theta}=l_{1} \frac{\lambda \sin (\theta)}{\left[1+\lambda^{2}+2 \lambda \cos (\theta)\right]^{1 / 2}}, \\
& \frac{d l_{4}}{d \theta}=l_{1} \frac{\lambda \sin (\theta)}{\left[1+\lambda^{2}-2 \lambda \cos (\theta)\right]^{1 / 2}} .
\end{aligned}
$$

In addition, we have the trivial deformations $d l_{1} / d \theta=0$ and $d l_{2} / d \theta=0$ associated with the inextensibility of the reinforcing fibers. Since the initial and final fiber angles are known, the total change in lengths $l_{3}$ and $l_{4}$ can be determined from these relationships. Unfortunately, since the orientation of these characteristic lengths may change throughout the deformation, the total length change cannot be directly related to the inertial strain components $\epsilon_{x x}$ and $\epsilon_{y y}$ except in the case where $\lambda=1$.

The inertial strain components can be calculated by segmenting the sample compression into differential steps and translating the characteristics into the inertial directions at each differential step. Alternatively, it can be shown that simplified strain dynamics actually still represent the more complex geometries, as we will discuss next.

\subsection{Special Case: Equal Fiber Lengths}

The geometric strain modeling is simplified drastically if the fiber ratio $\lambda=1$, meaning that fiber lengths $l_{1}$ and $l_{2}$ are equal to each other. For this case, no matter what the fiber angle $\theta$ is, or how much compression the basic element undergoes, the characteristic lengths $l_{3}$ and $l_{4}$ do not change orientation. This means that the overall change in the characteristic lengths can be related to the overall strain of the material in those directions. In terms of the initial and final fiber angles, the material strain components in the orthogonal $l_{3}$ and $l_{4}$ directions are,

$$
\epsilon_{l_{4}}=\left[\frac{1-\cos \left(\theta_{f}\right)}{1-\cos \left(\theta_{0}\right)}\right]^{1 / 2}-1, \quad \epsilon_{l 3}=\left[\frac{1+\cos \left(\theta_{f}\right)}{1+\cos \left(\theta_{0}\right)}\right]^{1 / 2}-1 .
$$

Substituting equation (4) for the final fiber angle, allows the strains in the principle axes to be calculated in terms of the initial fiber angle and the total compressive strain,

$$
\epsilon_{l 4}=\left[\frac{1-\left[1-\sin ^{2}\left(\theta_{0}\right) \frac{\left(\epsilon_{V}+1\right)^{2}}{\left(\epsilon_{z z}+1\right)^{2}}\right]^{1 / 2}}{1-\cos \left(\theta_{0}\right)}\right]^{1 / 2}-1, \epsilon_{l 3}=\left[\frac{1+\left[1-\sin ^{2}\left(\theta_{0}\right) \frac{\left(\epsilon_{V}+1\right)^{2}}{\left(\epsilon_{z z}+1\right)^{2}}\right]^{1 / 2}}{1+\cos \left(\theta_{0}\right)}\right]^{1 / 2}-1 .
$$


These material strains can then be related to the inertial strain components, $\epsilon_{x x}, \epsilon_{y y}$, and $\gamma_{x y}$, through a rotational strain transformation governed by the angle $\beta$, shown in figure 3 .

$$
\begin{aligned}
& \epsilon_{x x}=\epsilon_{l 4} \cos ^{2}\left(\frac{\pi-\theta}{2}-\beta\right)+\epsilon_{l 3} \sin ^{2}\left(\frac{\pi-\theta}{2}-\beta\right), \\
& \epsilon_{y y}=\epsilon_{l 4} \cos ^{2}(\theta / 2+\beta)+\epsilon_{l 3} \sin ^{2}(\theta / 2+\beta), \\
& \gamma_{x y}=\left(\epsilon_{y y}-\epsilon_{x x}\right) \sin (\pi-\theta-2 \beta) .
\end{aligned}
$$

For simplicity, all samples used in testing have an initial orientation set to $\beta_{0}=\left(\pi-\theta_{0}\right) / 2$ so that,

$$
\begin{aligned}
& \epsilon_{x x}=\epsilon_{l 4}=\left[\frac{1-\left[1-\sin ^{2}\left(\theta_{0}\right) \frac{\left(\epsilon_{V}+1\right)^{2}}{\left(\epsilon_{z z}+1\right)^{2}}\right]^{1 / 2}}{1-\cos \left(\theta_{0}\right)}\right]^{1 / 2}-1 \\
& \epsilon_{y y}=\epsilon_{l 3}=\left[\frac{1+\left[1-\sin ^{2}\left(\theta_{0}\right) \frac{\left(\epsilon_{V}+1\right)^{2}}{\left(\epsilon_{z z}+1\right)^{2}}\right]^{1 / 2}}{1+\cos \left(\theta_{0}\right)}\right]^{1 / 2}-1 \\
& \gamma_{x y}=0
\end{aligned}
$$

Here we showed that for the special case where fiber lengths are equal $(\lambda=1)$ the primary components of strain do not change direction, and therefore, can be solved analytically from the fiber angle dynamics. Next, we go on to show that these simplifications can be made even when the fiber lengths are not equal.

\subsection{Equivalence of Parallelagram Elements} deformation), despite the fact that $l_{2}^{\prime}$ does not lie on an actual reinforcing fiber, and the strain dynamics of the soft composite can be determined from $l_{3}^{\prime}$ and $l_{4}^{\prime}$ according to 8 . This means that the strain dynamics of a soft composite sheet is only dependent on the fiber angle $\theta$, and does not depend on the exact fiber lengths or length ratio. 


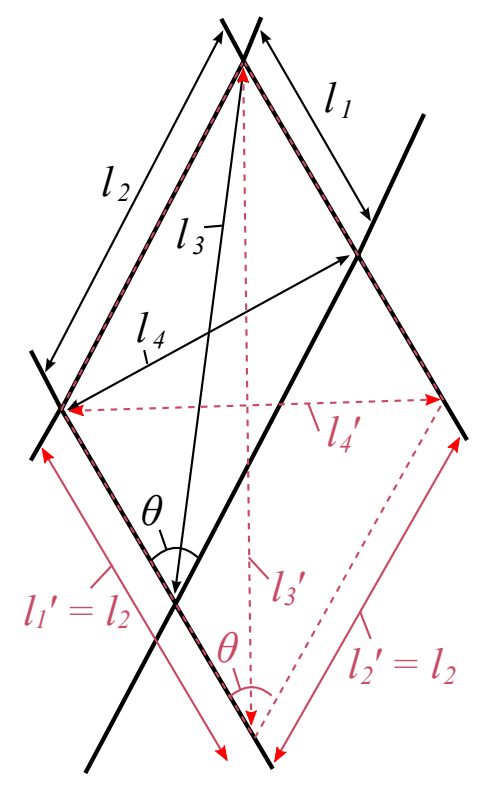

Figure 4: Diagram illustrating how a theoretical rhomboid element can be imposed on top of a parallelogram element with non-equal fiber lengths.

Here it is worth discussing the new deformation space of the sheet. An unreinforced elastomeric sheet has a truly infinite deformation space, and the 9 (6 for isotropic materials) principle components of strain can all vary independently, depending on the loading of the sample. With fiber reinforcement, the deformation of the sample is greatly restricted. As can be seen from equations $(9)$ and $\left(10\right.$ the planar strains $\epsilon_{x x}, \epsilon_{y y}$, and $\gamma_{x y}$ are all uniquely defined by the change in thickness, $\epsilon_{z z}$, and the material compressibility $\epsilon_{V}$. Therefore, equations $(9)$ and $(10)$ define the null space of deformations that satisfy the lack of extension in the fibers. Even though the possible deformations are now limited, if the goal is to drive planar deformation from transverse loading, $\epsilon_{x x}$ can be greatly increased without any additional planar loading. This is demonstrated in section 5

\section{Modeling Stiffness and Energetics}

\subsection{Elastic Modulus and Stress}

The approach we take here is to model the elastomeric matrix and fiber arrays as independent systems that interact through stresses in the planar directions that would be necessary to create the constrained deformations. The unreinforced elastomer is an isotropic, homogeneous material, meaning that compressing a sheet of the elastomer results in an outward strain in each of the planar directions as governed by the material's Poisson ratio. When the sheet is reinforced by fiber arrays, then the planar strains are instead governed by the fiber orientation, as described in the previous section. The stresses transfered between the fibers and elastomeric matrix in the planar directions can then be calculated as the stresses required to create the reinforced sample strains.

In tensorial notation, the relationship between any strain component and the different components of stress for an isotropic, homogeneous material is given by, $\epsilon_{i j}=\frac{1}{E}\left[\sigma_{i j}(1+\nu)-\nu \delta_{i j} \sigma_{k k}\right]$, [25], where $E$ is the elastic modulus of the material, $\sigma$ is the stress in the material, and $\delta$ is the Kronecker delta. Considering only normal stresses and strains 


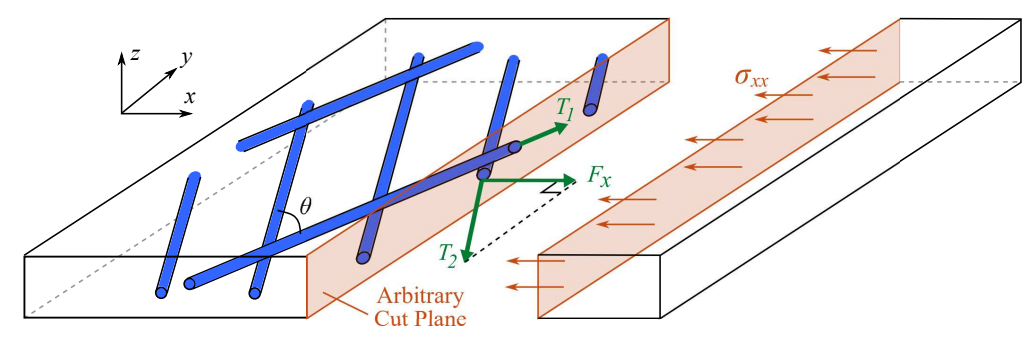

Figure 5: Diagram illustrating a hypothetical cut through a soft composite sheet, and the balance of forces between the normal component of tension in the reinforcing fibers and the material strain.

the dynamics for the homogeneous material can be written,

$$
\begin{aligned}
\epsilon_{x x} & =\frac{1}{E}\left[\sigma_{x x}-\nu\left(\sigma_{y y}+\sigma_{z z}\right)\right] ; \\
\epsilon_{y y} & =\frac{1}{E}\left[\sigma_{y y}-\nu\left(\sigma_{x x}+\sigma_{z z}\right)\right] ; \\
\epsilon_{z z} & =\frac{1}{E}\left[\sigma_{z z}-\nu\left(\sigma_{x x}+\sigma_{y y}\right)\right] .
\end{aligned}
$$

Here, the different options for modeling the stress strain relationships in soft composites become clear. One option is to treat the material as a non-isotropic composite which only requires a simplified version of (111) to describe the dynamics, since there is only an external stress in the $z$-direction. However, the elastic modulus, $E$, must then be modeled for the non-linear material, often done heuristically. Here we instead model the elastomeric modulus as an isotropic material, but include stresses in the planar directions exerted on the material by the reinforcing fibers needed to create the planar strains. During the deformation the reinforcing fibers are placed in tension, and first we will consider the general case where $l_{1} \neq l_{2}$; such that fibers aligned in the direction $\hat{e}_{l 1}$ have a different tension, $T_{1}$, than fibers aligned in the $\hat{e}_{l 2}$ direction with tension $T_{2}$, as shown in figure 5. By making a cut in the material in the $y-z$ or $x-z$ planes, the total forces transfered from the fibers to the elastomer are the product of the fiber tensions projected normal to the cut and the number of fibers. The planar stresses, $\sigma_{x x}$ and $\sigma_{y y}$, can then be calculated by the force balance between fibers and elastomer, and dividing the total fiber force by the cut area. The planar stresses in terms of fiber configuration and fiber tensions are

$$
\begin{aligned}
\sigma_{x x} & =\frac{-1}{h_{0} \sin \theta_{0}}\left[\frac{\cos \beta_{0}}{l_{1}} T_{1} \cos (\theta+\beta)+\frac{\cos \left(\theta_{0}+\beta_{0}\right)}{l_{2}} T_{2} \cos (\beta)\right] ; \\
\sigma_{y y} & =\frac{1}{h_{0} \sin \left(\theta_{0}\right)}\left[\frac{\sin \beta_{0}}{l_{1}} T_{1} \sin (\theta+\beta)+\frac{\sin \left(\theta_{0}+\beta_{0}\right)}{l_{2}} T_{2} \sin (\beta)\right] .
\end{aligned}
$$

Inserting equations (12) and (10) into (11) for the planar stress and strain terms, respectively, provides a system of three equations to solve the three unknown forcing terms, $\sigma_{z z}, T_{1}$, and $T_{2}$ in the general system. For the remainder of the analysis, for simplicity, we will restrict ourselves to the case where $l_{1}=l_{2}$ and $\beta=(\pi-\theta) / 2$, such that all fibers have the same tension, $T$. For this simplification, the planar stress terms can be rewritten,

$$
\begin{aligned}
\sigma_{x x} & =C_{1} \sqrt{1-\cos (\theta)} T, & C_{1} & =\frac{\sqrt{2} \sin \left(\frac{\theta_{0}}{2}\right)}{h_{0} \sin \left(\theta_{0}\right) l_{1}} ; \\
\sigma_{y y} & =C_{2} \sqrt{1+\cos (\theta)} T, & C_{2} & =\frac{\sqrt{2} \cos \left(\frac{\theta_{0}}{2}\right)}{h_{0} \sin \left(\theta_{0}\right) l_{1}} .
\end{aligned}
$$


For these conditions, the forcing terms are defined by,

$$
\begin{aligned}
& T=\frac{E\left(\left[\frac{1-\cos (\theta)}{1-\cos \left(\theta_{0}\right)}\right]^{1 / 2}-1+\nu \epsilon_{z z}\right)}{C_{1}\left(1-\nu^{2}\right) \sqrt{1-\cos (\theta)}-C_{2} \nu(1+\nu) \sqrt{1+\cos (\theta)}} \\
& \sigma_{z z}=E \epsilon_{z z}+\nu\left(C_{1} \sqrt{1-\cos (\theta)}+C_{2} \sqrt{1+\cos (\theta)}\right) T
\end{aligned}
$$

and $\cos (\theta)$ is a function of the $z$ strain and volumetric strain,

$$
\cos (\theta)=\sqrt{1-\sin ^{2}\left(\theta_{0}\right)\left(1+\epsilon_{V}\right)^{2} /\left(1+\epsilon_{z z}\right)^{2}}
$$

In this derivation it is assumed that the stress and strain of the elastomeric matrix have a linear relationship over the entire range of compressions. Hence, the elastic modulus, $E$, has a constant value. It will be shown in section 5 that the matrix stress-strain relationship is only linear for compressive strains smaller than approximately 0.5 , after which the stress increases at an exponential rate. Although the present investigation does not necessitate higher order modeling of the stress-strain relationship, the elastic modulus in equations (11) and 14 can easily be replaced with some function of the compressive and volumetric strains $E\left(\epsilon_{z z}, \epsilon_{V}\right)$ without affecting the general procedure.

\subsection{Strain Energy and Deformation Work}

The work required to enact a given deformation, ignoring any possible loading associated with specific applications, is equal to the potential energy stored in the soft composite during the deformation process. Since the force interaction between the fibers and the elastomeric matrix has already been defined, we will similarly calculate the necessary deformation work as the sum of the strain energy stored in the elastomer and fiber systems. For the purposes of this derivation we will again be assuming that the fiber lengths are equal, $l_{1}=l_{2}$, and that $\beta_{0}=\left(\pi-\theta_{0}\right) / 2$ such that all fibers have the same tension, $T$, and the characteristic strains of the fiber element line up with the inertial axes (10).

First consider the elastomeric matrix. In the absence of any shear stresses, the potential energy stored in a differential element of the elastomer volume is equal to the sum of the three normal components of stress integrated over their respective strains [25].

$$
\begin{aligned}
d U_{\text {matrix }} & =\left(\int_{0}^{\epsilon_{z z}} \sigma_{z z} d \tilde{\epsilon}_{z z}+\int_{0}^{\epsilon_{x x}} \sigma_{x x} d \tilde{\epsilon}_{x x}+\int_{0}^{\epsilon_{y y}} \sigma_{y y} d \tilde{\epsilon}_{y y}\right) d V, \\
& =\int_{0}^{\epsilon_{z z}}\left(\sigma_{z z}+\sigma_{x x} \frac{\partial \epsilon_{x x}}{\partial \epsilon_{z z}}+\sigma_{y y} \frac{\partial \epsilon_{y y}}{\partial \epsilon_{z z}}\right) d \tilde{\epsilon}_{z z} d V
\end{aligned}
$$

In this energy equation, $\tilde{\epsilon}_{x x}, \tilde{\epsilon}_{y y}$, and $\tilde{\epsilon}_{z z}$ are dummy variables for the principle strains, and the three integral terms are combined under a single integration by changing the variable of integration utilizing the strain relationships $d \epsilon_{x x} / d \epsilon_{z z}$ and $d \epsilon_{y y} / d \epsilon_{z z}$ which can be calculated from 10 ,

$$
\begin{aligned}
\frac{\partial \epsilon_{x x}}{\partial \epsilon_{z z}} & =\frac{-\sin ^{2}\left(\theta_{0}\right)\left(1+\epsilon_{V}\right)^{2}}{2\left[\cos ^{2}(\theta)(1-\cos (\theta))\left(1-\cos \left(\theta_{0}\right)\right)\right]^{1 / 2}\left(1+\epsilon_{z z}\right)^{3}}, \\
\frac{\partial \epsilon_{y y}}{\partial \epsilon_{z z}} & =\frac{\sin ^{2}\left(\theta_{0}\right)\left(1+\epsilon_{V}\right)^{2}}{2\left[\cos ^{2}(\theta)(1+\cos (\theta))\left(1-\cos \left(\theta_{0}\right)\right)\right]^{1 / 2}\left(1+\epsilon_{z z}\right)^{3}} .
\end{aligned}
$$

Fortunately, the terms for stresses from the fibers in the planar directions cancel out, and the strain energy for the differential element can be rewritten as simply,

$$
d U_{\text {matrix }}=\left(\int_{0}^{\epsilon_{z z}} E \epsilon_{z z}+\nu\left[C_{1} \sqrt{1-\cos (\theta)}+C_{2} \sqrt{1+\cos (\theta)}\right] T d \tilde{\epsilon}_{z z}\right) d V
$$


where $T, C_{1}, C_{2}$, and $\cos (\theta)$ were all defined previously as functions of $\epsilon_{z z}$ and $\epsilon_{V}$. It can be understood in this equation that the first term in the integrand, $E \epsilon_{z z}$, corresponds to the potential energy storage that would be found in an unconstrained homogeneous sample. The additional term in the integrand is the additional energy required to deform the reinforced material, corresponding to the increase in stiffness associated with the coupled planar strains.

Now consider the fiber arrays themselves. Clearly, a much simpler system, since the tension force, $T$, extends in

the direction of the fiber axis. It should be noted that the fiber was assumed to be inextensible since they have a much higher stiffness than the elastomer matrix, so that the strain of the fibers would be much less than the strain in the elastomer. The high tensile modulus also means that there is appreciable energy stored in the fibers, despite the very low strain.

We assume that the fibers have a linear stress/strain relationship in this low strain range with a constant modulus, $E_{f}$. Therefore, the total energy stored in the fibers is half the product of the stress, strain, total fiber length, and number of fibers. This can be rewritten in terms of the tension and initial configuration,

$$
U_{\text {fibers }}=\frac{W_{0} L_{0}}{l_{1} E_{f}\left(\pi r_{f}^{2}\right)^{2} \sin \left(\theta_{0}\right)} T^{2},
$$

where $r_{f}$ is the radius of the fiber.

The total work required to create the deformation in the soft composite is the sum of the strain energy of the fibers and elastomeric matrix, $U=U_{\text {matrix }}+U_{\text {fibers }}$.

\section{Materials and Methods}

We fabricated a set of soft composite sheets constructed out of Maxelast C4900 (from APS elastomers), a soft thermo-plastic elastomer (TPE). Embedded within the rubber is an array of cotton yarn fibers that can be considered 
Table 1: Useful composite parameters and states are defined for cases where loading is applied in either the $\mathrm{x}$ or $\mathrm{y}$-directions. Consistent with previous definitions $\theta$ is the fiber angle at any time in the deformation, $\epsilon_{z z}$ is the resulting strain in the z-direction, $T$ is the tension in the fibers, and $\sigma_{x x, y y}$ is the stress needed to create planar strains either $\epsilon_{x x}$ or $\epsilon_{y y}$

\begin{tabular}{|c|c|c|}
\hline & Loading in x-direction & Loading in y-direction \\
\hline$\theta$ & $\operatorname{acos}\left[1-\left(\epsilon_{x x}+1\right)^{2}\left(1-\cos \left(\theta_{0}\right)\right)\right]$ & $\operatorname{acos}\left[\left(\epsilon_{y y}+1\right)^{2}\left(1+\cos \left(\theta_{0}\right)\right)-1\right]$ \\
\hline$\epsilon_{z z}$ & $\frac{1+\epsilon_{V}}{1+\epsilon_{x x}} \sqrt{1+\cos \left(\theta_{0}\right)}$ & $\frac{\left(1+\epsilon_{V}\right) \sin \left(\theta_{0}\right)}{\sqrt{2-\left(\epsilon_{y y}+1\right)^{2}\left(1+\cos \left(\theta_{0}\right)\right)}}$ \\
\hline$T$ & $-\frac{E\left(1+\epsilon_{V}\right)}{\nu\left(1+\epsilon_{x x}\right)} \frac{E\left(1+\epsilon_{V}\right) \sin \left(\theta_{0}\right)}{C_{1} \sqrt{1-\cos (\theta)}+C_{2} \sqrt{1+\cos (\theta)}}$ & $-\frac{\sqrt{1+\cos \left(\theta_{0}\right)}}{\nu\left[C_{1}(1-\cos (\theta))+C_{2} \sqrt{1-\cos ^{2}(\theta)}\right]}$ \\
\hline$\sigma_{x x / y y}$ & $C_{1} \sqrt{1-\cos (\theta)} T$ & $C_{2} \sqrt{1+\cos (\theta)} T$ \\
\hline
\end{tabular}

to be inextensible along their length, as their tensile strength, $1.9 \mathrm{GPa}$, is orders of magnitude higher than that of the elastomer matrix, $150 \mathrm{kPa}$. Each sample is $63.5 \mathrm{~mm}(2.5 ")$ square by $6.4 \mathrm{~mm}(0.25 ")$ thick. Each sample is created by first stretching the fibers out on custom loom, then inserting them between two halves of a square mold. Finally the mold is injected with the elastomer matrix with a MediumMachinery Mold Press. A summary of all samples used in testing, and their fiber orientations, is provided in table 2 .

The samples are then compressed to a consistent thickness using an apparatus that consists of an arbor press and a custom compression jig, as shown in figure 6a. The purpose of the compression jig is to spread the force of the press over the entire soft composite sample, while at the same time providing visibility of the planar deformation of the sample, and is shown in more detail in the CAD image in figure 6b. The jig consists of two $127 \times 127 \times 12.8 \mathrm{~mm}$ $(5 \times 5 \times 0.5$ in $)$ rectangular plates. The top plate is made out of aluminum and bottom plate is made out of acrylic. The aluminum plate has a square pocket cut into it which fits to the press foot in order to prevent excessive movement during testing. Four threaded rods were fastened to the aluminum plate and pressed onto a buffer plate, or washer, that is set on top of the acrylic plate in order to spread the load and reduce the risk of cracking the brittle plastic. The rods and the buffer plate transfer the force from the press onto the clear acrylic plate which compresses the test samples. A mirror is suspended at an angle within the compression jig so that the top view of the samples can be seen from the front.

The entire frame is set on top of each sample and pictures are taken with a high resolution Nikon SLR of the sample in both the compressed and uncompressed state. A ruler is fixed to the bottom acrylic plate in both the horizontal and vertical directions, in case there is any parallax from the angled mirror to be corrected. Three sets of rigid rectangular struts with thicknesses of $5.1 \mathrm{~mm}(0.2 \mathrm{in}), 4.8 \mathrm{~mm}\left(3 / 16^{\prime \prime}\right)$, and $4.1 \mathrm{~mm}(0.16 \mathrm{in})$ are set on either side of the sample. The struts guarantee that the compression jig settles at a consistent final thickness and guarantee uniform compressive strain over the entire sample. Here it should be noted that some of the samples have fiber angles too high to reach these standard final thicknesses. As the sample compresses and the area of the basic fiber element increases, the fiber angle also increases. When the fiber angle reaches $90^{\circ}(\pi / 2)$, the area of the basic element is maximized and further compression is only possible through stretching of the matrix above and below the fiber plane, which contradicts the uniform compression strain assumption of the model. Therefore, samples with sufficiently high starting angle had reduced final compression, as is reflected in table 2 and discussed further in section 5 
Table 2: Summary of all the experimental test samples. All samples have a starting thickness of $6.4 \mathrm{~mm}(0.25 ")$ and length ratio $\lambda=1$, and the fibers run through the center of the sample thickness.

\begin{tabular}{cccccc}
\hline \hline Sample & $\theta_{0}$ & $\theta_{f}$ & $\theta_{f}$ & $\epsilon_{x x}$ & $\epsilon_{x x}$ \\
& & $\left(@ \epsilon_{z z}=-0.2\right)$ & $\left(@ \epsilon_{z z}=-0.35\right)$ & $\left(@ \epsilon_{z z}=-0.2\right)$ & $\left(@ \epsilon_{z z}=-0.35\right)$ \\
\hline 1 & $19.9^{\circ} \pm 0.9^{\circ}$ & $23.1^{\circ} \pm 0.6^{\circ}$ & $27.8^{\circ} \pm 0.5^{\circ}$ & $0.20 \pm 0.04$ & $0.40 \pm 0.07$ \\
2 & $26.4^{\circ} \pm 0.8^{\circ}$ & $31.5^{\circ} \pm 0.7^{\circ}$ & $38.6^{\circ} \pm 1.2^{\circ}$ & $0.23 \pm 0.06$ & $0.52 \pm 0.06$ \\
3 & $29.3^{\circ} \pm 1.5^{\circ}$ & $37.7^{\circ} \pm 1.4^{\circ}$ & $43.5^{\circ} \pm 0.8^{\circ}$ & $0.23 \pm 0.07$ & $0.47 \pm 0.11$ \\
4 & $35.2^{\circ} \pm 1.3^{\circ}$ & $43.4^{\circ} \pm 0.5^{\circ}$ & $49.2^{\circ} \pm 1.4^{\circ}$ & $0.25 \pm 0.05$ & $0.43 \pm 0.06$ \\
5 & $39.8^{\circ} \pm 1.4^{\circ}$ & $48.5^{\circ} \pm 2.0^{\circ}$ & $58.9^{\circ} \pm 1.5^{\circ}$ & $0.25 \pm 0.05$ & $0.52 \pm 0.05$ \\
6 & $42.2^{\circ} \pm 1.2^{\circ}$ & $55.0^{\circ} \pm 1.5^{\circ}$ & $62.6^{\circ} \pm 1.1^{\circ}$ & $0.33 \pm 0.03$ & $0.50 \pm 0.06$ \\
7 & $45.5^{\circ} \pm 1.5^{\circ}$ & $59.1^{\circ} \pm 1.5^{\circ}$ & $68.0^{\circ} \pm 1.4^{\circ}$ & $0.27 \pm 0.05$ & $0.50 \pm 0.05$ \\
8 & $56.0^{\circ} \pm 2.4^{\circ}$ & $76.0^{\circ} \pm 1.7^{\circ}$ & $79.6^{\circ} \pm 2.2^{\circ}$ & $0.35 \pm 0.06$ & $0.46 \pm 0.07$ \\
9 & $61.3^{\circ} \pm 1.7^{\circ}$ & $79.4^{\circ} \pm 1.5^{\circ}$ & $88.4^{\circ} \pm 1.9^{\circ}$ & $0.33 \pm 0.08$ & $0.40 \pm 0.08$ \\
10 & $67.8^{\circ} \pm 2.7^{\circ}$ & $84.6^{\circ} \pm 3.6^{\circ}$ & $88.2^{\circ} \pm 2.6^{\circ}$ & $0.31 \pm 0.08$ & $0.31 \pm 0.07$ \\
11 & $73.0^{\circ} \pm 1.6^{\circ}$ & $87.8^{\circ} \pm 2.3^{\circ}$ & $90.4^{\circ} \pm 2.4^{\circ}$ & $0.25 \pm 0.05$ & $0.25 \pm 0.05$ \\
12 & $76.0^{\circ} \pm 4.0^{\circ}$ & $89.7^{\circ} \pm 1.6^{\circ}$ & $91.2^{\circ} \pm 2.2^{\circ}$ & $0.23 \pm 0.08$ & $0.23 \pm 0.08$ \\
13 & $84.0^{\circ} \pm 1.5^{\circ}$ & $90.4^{\circ} \pm 2.8^{\circ}$ & $92.8^{\circ} \pm 5.1^{\circ}$ & $0.13 \pm 0.05$ & $0.13 \pm 0.05$
\end{tabular}

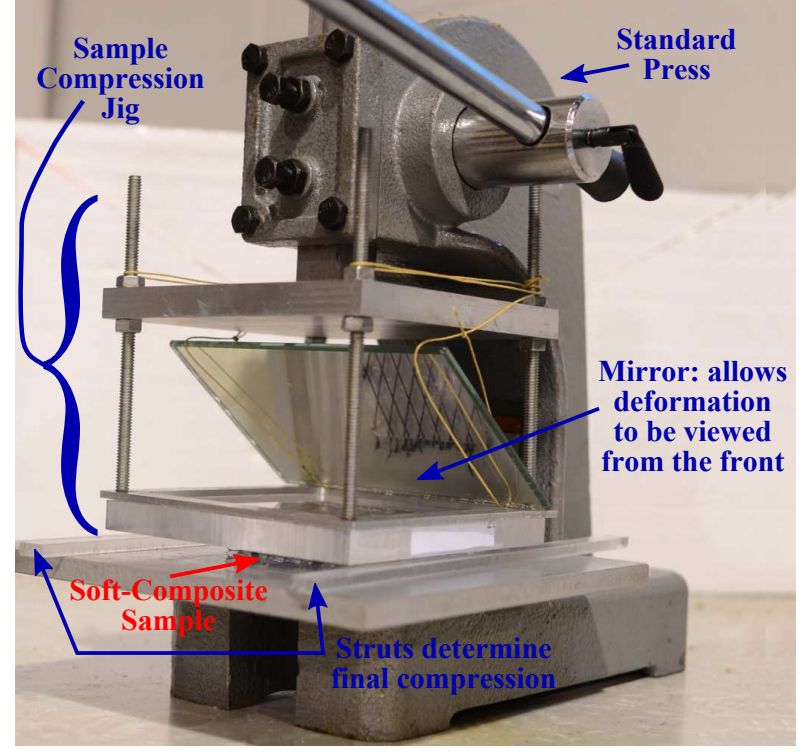

(a)

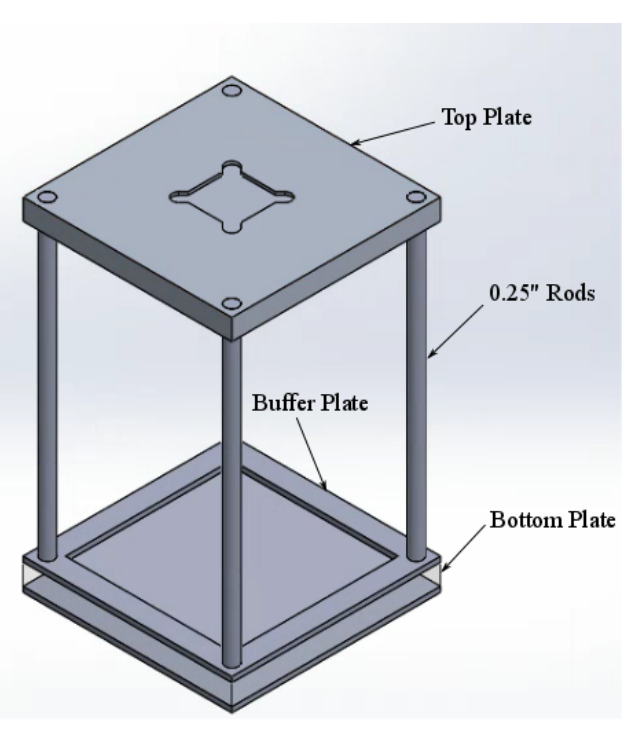

(b)

Figure 6: The total soft composite sample compression apparatus is shown (a) along with CAD illustrations of the compression jig (b). 

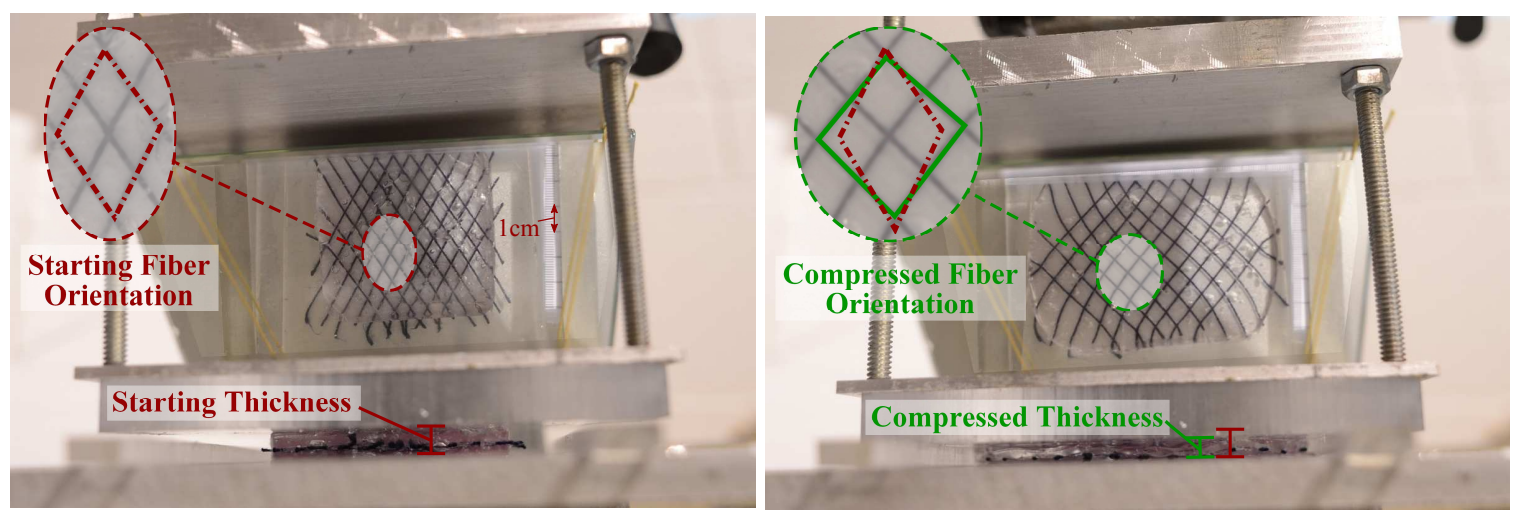

Figure 7: A sample (sample 5 from table 2 is shown in the compression setup both in the resting state (left) and under compression (right) with a compressive strain $\epsilon_{z z}=0.23$. The actual sample can be seen at the bottom of each image being pressed between two plates, and above is the mirror reflection of the top view. A small section of the sample in each configuration has been enlarged to show the change in fiber orientation during compression.

Prior to compression, the contact faces of the samples were lubricated with WD-40 in order to minimize the friction between the elastomer and aluminum and acrylic plates, respectively. This helps to ensure uniform compressive strain. The images of compressed and uncompressed samples were analyzed by identifying intersections of the fiber arrays,

and calculating the geometry of several elements in each sample. The rulers fixed to the top acrylic plate were used to calculate pixel scaling in the horizontal and vertical directions independently. Nevertheless the scaling varied by less than $1 \%$ between the different directions. Example images of a resting and compressed sample in the compression setup are shown in figure 7 A small section of the sample is enlarged in this figure to emphasize the change in fiber angle of a basic elements in the sample.

The compression force from the press is transmitted to the compression jig through a Futek LLB300 load cell to measure the compression force acting on the sample, which divided by the sample area is the compressive stress. When making stress/strain measurements of the soft composite samples, the struts are removed (since compression forces would be translated through those struts) and the strain is instead measured with a linear potentiometer also attached to the compression jig.

\section{Results}

\subsection{Volumetric Strain and Compression}

In order to model the stress, strain, and energy dynamics in the soft composite sheets, a relationship between strains in a primary direction and the resulting strains in the transverse directions must be known. Typically this relationship is captured by a single term, the Poisson ratio, which is coupled to the compressibility of the material. Given the large strains that will be experienced, the Poisson ratio cannot be treated as a constant, but instead a function of the loading. Typically stiffer materials result in higher compression, but the highly deformable elastomer, which in many ways acts as a liquid, experiences very little compression. Therefore, we will characterize the volumetric strain, and calculate the Poisson Ratio from (2).

The volumetric strain is assumed to be a function of the driving strain in the $z$-direction and the fiber angle. Furthermore, the volumetric strain must vanish as $\epsilon_{z z}$ drops to zero, and must be negative regardless of the direction 


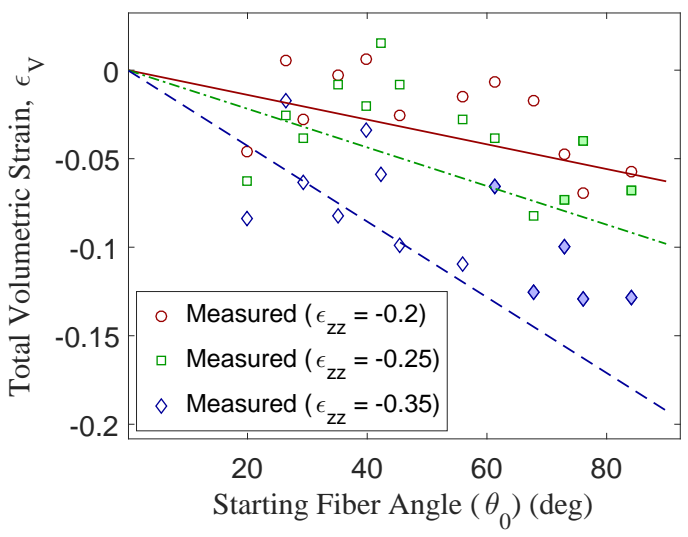

Figure 8: The compressibility of the soft composite sheets is characterized by the volumetric strains for different fiber reinforcement angles and the three different levels of $z$ strain. Also shown are the volumetric strains predicted by 20 , assuming that material compressibility is proportional to the $z$ strain energy and the fiber angle. The solid line corresponds to $\epsilon_{z z}=-0.2$, the dashed line to $\epsilon_{z z}=-0.25$ and the dashed-dotted line to $\epsilon_{z z}=-0.35$, respectively.

of $\epsilon_{z z}$. The simplest representation to capture these qualities is,

$$
\epsilon_{V}=-\theta_{0} \epsilon_{z z}^{2}
$$

which although simple, is a surprisingly accurate approximation. Figure 8 shows the volumetric strain measured in the test samples at all three levels of compression and for all the initial fiber angles tested. The significant fluctuations in the measured volumetric strains is mostly due to the fact that the measured strains are very small, many remaining below 5\%. The figure also shows the volumetric strain predicted by $(20)$, which is seen to capture the general trend in material compression despite the large noise in the experimental data.

\subsection{Fiber Angle Dynamics}

As the soft composite sheet thickness is compressed, the planar area must increase to conserve volume. Since the reinforcing fibers extend negligibly compared to the deformation of the elastomer, the increase in volume can only be accounted for by increasing the fiber angle. Figure 9 shows the final fiber angle of all the test samples after compression with respect to their original fiber angle. Also shown is the final fiber angle predicted by equation (4) using (20) to approximate the volumetric strain, given the initial fiber angle, $\theta_{0}$, and compressive strain, $\epsilon_{z z}$. It can be seen in this figure that there is excellent agreement between the predicted and actual final fiber angles, validating the geometric modeling of the soft composite sheet.

As we mentioned in previous sections there is a maximum possible compressive strain for any starting fiber angle, corresponding to the fiber angle reaching the critical value, $\theta=90^{\circ}(\pi / 2)$, where planar area can no longer increase. At a starting angle of $\theta_{0} \approx 50^{\circ}$ the maximum compressive strain is reached in the experiment, and all of the samples with higher starting angles maintain the maximum final fiber angle. As the compressed fiber angle approaches this maximum value, the actual final angle drops slightly below the predicted fiber angle due to minor stretching in the reinforcing fibers. The errors due to fiber stretching increase with the increased compressive loading, but for all cases remain fairly low.

Next we look into how reorienting the fiber array affects the strain (deformation) dynamics of the soft composites. 


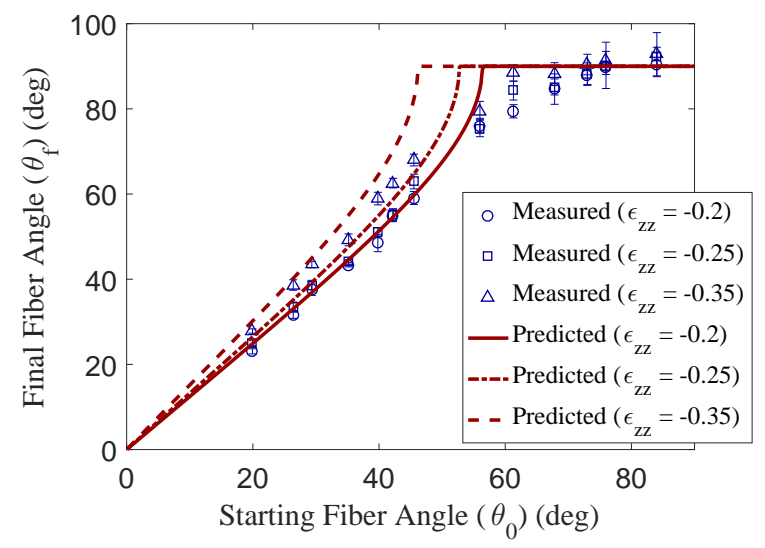

Figure 9: The final fiber angle vs. the starting fiber angle is shown for all soft composite samples after compressions of $\epsilon_{z z}=0.2, \epsilon_{z z}=0.25$, and $\epsilon_{z z}=0.35$ have been applied. Error bars indicate the standard deviation of measured fiber angles. The final fiber angles predicted by (4) with $\epsilon_{V}$ predicted by 20, are also shown.

\subsection{Strain Dynamics}

If compression of soft elastomer sheets is applied for soft actuation purposes, strain will be desired in one planar direction, and the other planar direction is inconsequential. In these situations the goal of the fiber reinforcement is to maximize the strain in the desired planar direction and minimize the strain in the orthogonal planar direction. By adding straight reinforcing fibers the strain in the orthogonal direction can be held to zero increasing the strain in the desired direction. One premise of this study is that the strain in the desired direction can be increased even further, by reinforcing with an angled array of reinforcing fibers, resulting in negative strain in the planar direction orthogonal to the desired strain.

The maximum compressive strain of a sample, which results in final fiber angles $\theta=90^{\circ}(\pi / 2)$, is a function of the initial fiber angle and volumetric strain at the critical loading (5). The theoretical resulting planar strain, $\epsilon_{x x}$, of soft composite samples with various reinforcing fiber orientations compressed to their limit is plotted in figure 10 , This figure also contains the planar strains of the test samples compressed experimentally, and the predicted strain at the three compression levels.

All the test samples were compressed to $\epsilon_{z z}=-0.2, \epsilon_{z z}=-0.25$, and $\epsilon_{z z}=-0.35$, except for those samples that reach the maximum fiber angle, at which point the compression is terminated. The resulting strains in the desired $\left(\epsilon_{x x}\right)$ direction are plotted in figure 10 . Since the $z$ strain must be reduced after the critical fiber angle is reached, the desired planar strain is normalized by the $z$ strain to allow a valid comparison for the high fiber angle cases. The normalized desired strain, $-\epsilon_{x x} / \epsilon_{z z}$, calculated by $\sqrt{10}$ is also plotted in figure 10 . It can be seen that the strain modeling is quite accurate, but the actual normalized strains at high fiber angles tend to be larger than predicted due to the fiber stretching.

As is shown in figure 10, the maximum possible normalized planar strain blows up at the two ends of the fiber angle spectrum $\theta_{0}=0$ and $\theta_{0}=90^{\circ}(\pi / 2)$. For low fiber angles this is largely due to an inconsistency in the range of compressive strains vs. the range of tensile strains for highly deformable materials. Consider a soft composite sheet with $\theta=0$, meaning that all the fibers are aligned parallel to the $y$-axis. No matter how far the sheet is compressed, the fibers will always remain in this parallel orientation, so for this case there is no limit to the compressive strain 


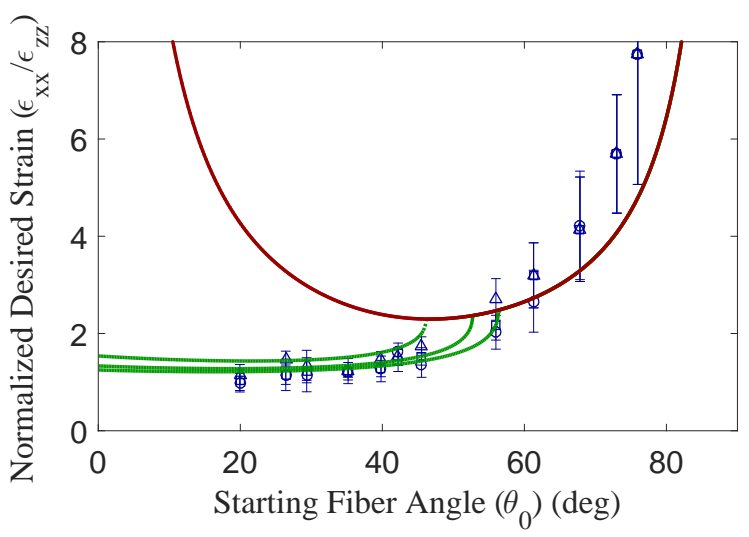

(a)

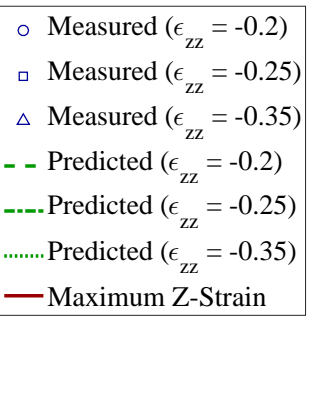

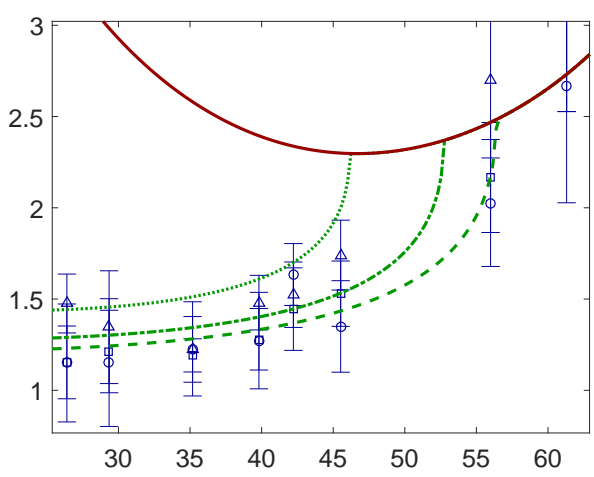

(b)

Figure 10: Desired planar strain $\epsilon_{x x}$ normalized by the compressive strain driving deformation $\epsilon_{z z}$ is plotted against the starting fiber angle, showing significant normalized strains at the higher starting fiber angles, and showing good agreement with the predicted normalized strains. The full range is shown in (a) and an enlarged inset showing the dynamic region where constant strains intersect with the maximum in (b). The solid red line depicts the theoretical maximum normalized strain 5 and the 3 green dashed lines represent the normalized strains predicted by 10 for the different $z$ strain cases.

due to reaching a critical fiber angle. The theoretical limit to the compressive strain in this case corresponds to the sheet thickness being reduced to zero, $\epsilon_{z z}=-1$. If such a compression is exerted, then the strain in the $x$-direction becomes infinite. There is an inconsistency since the compressive strain ranges from 0 to -1 , while the planar strain ranges from 0 to $\infty$. For smaller (realistically attainable) values of compressive strain, the normalized planar strain of a sheet with parallel reinforcing fibers is $\approx 1$. This is indicated by the values of normalized strain for the curves corresponding to $\epsilon_{z z}=-0.2,-0.25$, and -0.35 , which is a more representative value of the normalized strain at $\theta=0$.

On the other end of the spectrum, as the fiber angle approaches $\theta=\pi / 2$ the geometric constraints on the planar deformation cause the strain in the $y$-direction to become negative, which by conservation of volume increases the strain in the desired $x$-direction. In this sense the higher normalized strain of the high fiber angles, does correlate to a much larger deformation in the desired direction than is imposed in the $z$-direction. For all three curves representing constant compressive strain, it can be seen that the desired strain steadily increases with increasing initial fiber angle until the compressive strain results in the critical maximum fiber angle. At this point any higher fiber angle cannot support such high compressive strains, and the desired strain must then follow the maximum strain trend, further increasing the normalized planar strain, but reducing the $z$ strain. It can be seen that the soft composite samples used for testing matched these trends throughout the range of fiber angles tested, producing a strain in the desired direction as much as eight times the compressive strain driving deformation. This corresponds to 16 times the strain in $x$-direction that would be produced by an unreinforced sample under the same reduction in thickness.

In general the low fiber angles allow for maximum possible absolute strains in the desired planar direction, but benefit little from negative strains in the orthogonal planar direction. Contrarily, high fiber angles generally result in higher normalized strains in the desired direction because of the significant negative strains in the orthogonal planar direction, but are limited to smaller absolute strains possible before the critical fiber angle is reached.

The normalized planar strain has a physical meaning beyond just allowing consistent comparison of resulting planar 


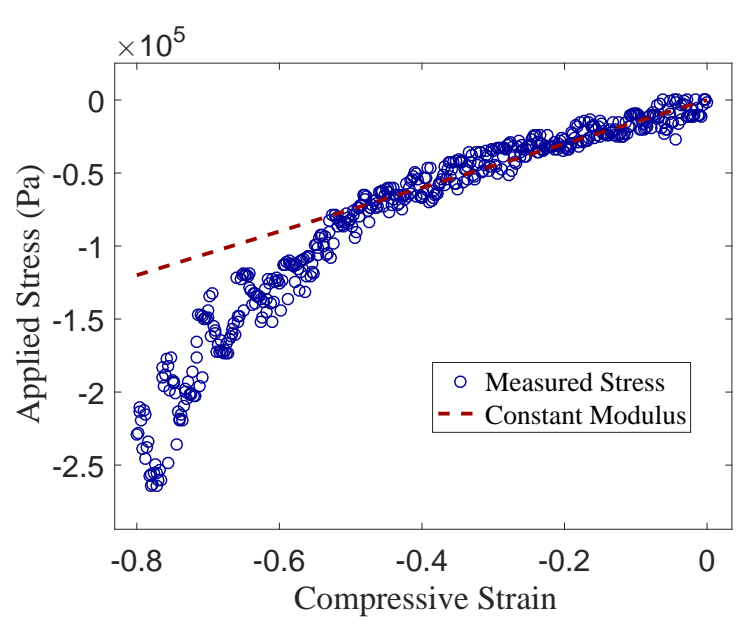

(a)

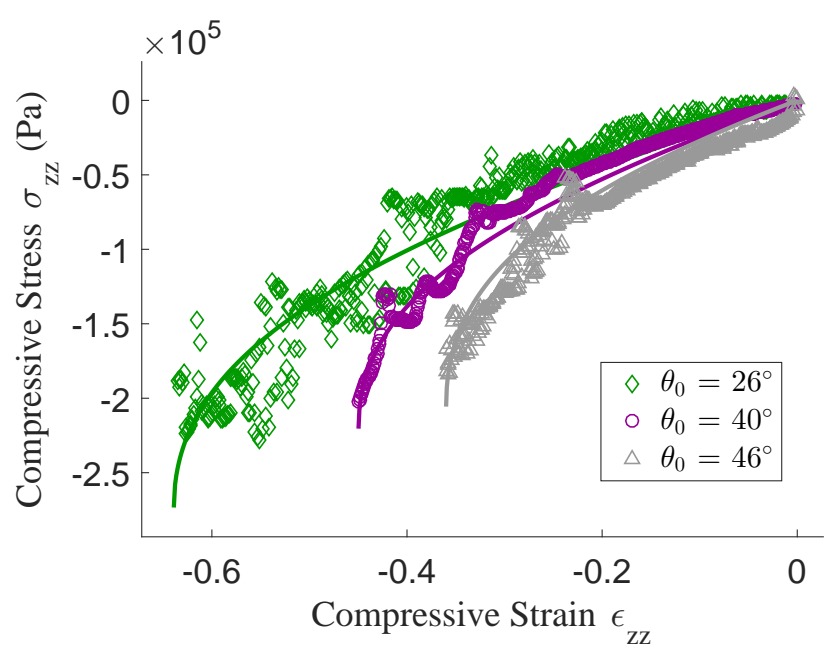

(b)

Figure 11: Stress strain relationship of the elastomeric matrix without any reinforcing fibers (a) and stress strain relationship for the soft composite sheets with various fiber angles. The dashed line in (a) shows a constant modulus of $E=150 \mathrm{kPa}$, and the solid lines in (b) are calculated for a soft composite sheet from 14 assuming $\epsilon_{V}$ of the form given by 20 .

is compressed with muscles or muscle-like soft actuators, the work put into the sheet is equal to the elastic spring force of the material integrated over the distance that the sheet is compressed, and therefore, scales with the strain in the $z$-direction. Since the desired output is the deformation in the $x$-direction, then the normalized planar strain can be considered an indicator of desirable deformations. In the next subsections we validate the modeling for soft composite stress-strain relationships, and investigate the effect of fiber orientation on total energy required to create a deformation.

\subsection{Compression Force and Variable Stiffening}

As we discussed in section 3 , the elastomer matrix is considered to be an isotropic homogeneous material that interacts with the reinforcing fibers through normal stresses applied throughout its volume. Therefore, it makes sense to first look at the elastic properties of the TPE without any reinforcement. A non-reinforced sample was created with the same overall shape and size of the soft composite samples, and the applied stress was measured throughout compression along with the resulting compressive strain. Figure 11] shows the stress-strain relationship for the unreinforced sample, averaged over multiple trials. It can be seen in this figure that over a fairly large range of the deformation, up to $\epsilon_{z z} \approx 0.5$, the stress-strain relationship remains linear with a corresponding TPE elastic modulus of $E=150 \mathrm{kPa}$, which is also plotted in figure 117 for comparison.

Similarly the soft composite samples with varying fiber reinforcement orientations were compressed while simultaneously measuring compressive stress and strain. Given the range of compressive strain, the resulting fiber tension and compressive stress were calculated from (14) using the elastic modulus just determined, and the volumetric strain approximation provided by 200 .

Figure 11b shows the measured stress strain relationship for three composite samples along with the stress strain relationship predicted by the fiber interaction modeling. These three samples, samples 2, 5 , and 7 in table 2 , are chosen because they span the fiber orientation range without cluttering the figure, but it should be noted that the 


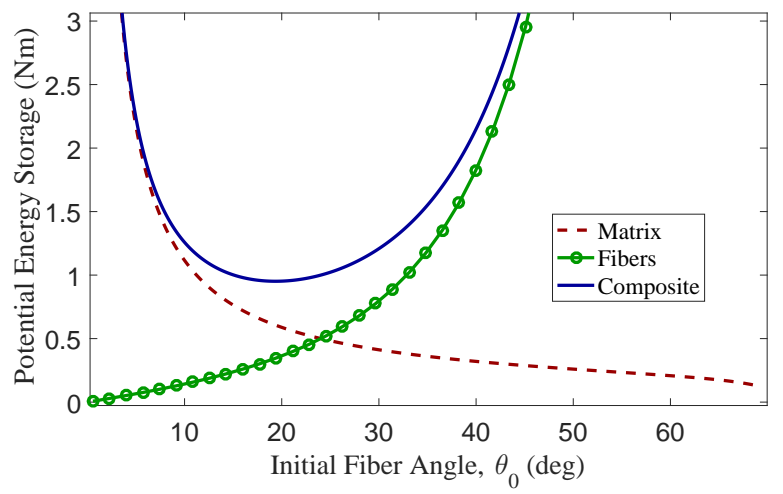

Figure 12: Potential energy stored in different components of the soft composite sheets in order to create a planar strain of 0.25 as a function of the starting fiber angle.

modeling was similarly accurate for all other samples. It can be seen that the modeling predicts the stress required to compress the samples with excellent accuracy, which validates the methodology that the elastomeric matrix and fiber arrays can be considered independent systems that interact through planar stresses throughout the volume.

It can also be observed that, as predicted, the composite sample stiffness increases with both the initial fiber angle and with the compressive strain that induces fiber reorientation. This is evident by the fact that the samples with higher initial fiber angle require more stress to create the same compressive strain, and by the non-linear stress-strain relationship even in the small strain region. Although an increased fiber angle will require a larger stress to create a given $z$ strain, it will also result in larger induced planar strains. In the next section we look into the potential energy storage in the material to determine whether, from a design point of view, the increased stiffness is justified by the increased output in the desired direction.

\subsection{Elastic Potential Energy and Optimal Fiber Orientation}

It was shown in the previous sections that the orientation of arrays of antagonistic reinforcing fibers significantly affect the stress and strain dynamics of soft composite sheets. More specifically, strain in one specific planar direction can be increased significantly, for a sheet under compression, while the strain in the other planar direction actually becomes negative. This phenomenon is clearly advantageous for applications like EAP actuation where extension of soft material is achieved through compression in a transverse direction. However, as was also shown, the increase in planar strain comes at the cost of an increase in material stiffness. Here we address the question as to whether the increase in desired planar strain decreases overall work required to create a given deformation.

The total energy required to create a given deformation is the sum of the potential energy stored in the elastomeric matrix (18) and the potential energy stored in the fiber system (19). The integral equation that describes potential energy storage in the elastomeric matrix cannot be easily solved analytically, so we approximated this term numerically. For this process, first a desired planar strain is prescribed, then the compressive strain required to reach that planar strain is determined based on the initial fiber orientation. Taking differential steps in the sample thickness compression the stresses, fiber orientations, and rate of potential energy storage are calculated for each differential step. The numerical iteration and step size determination were done by a Matlab integrator (ODE45).

As an example consider the case where a compression is needed to create a desired planar stress of 0.25 . The 


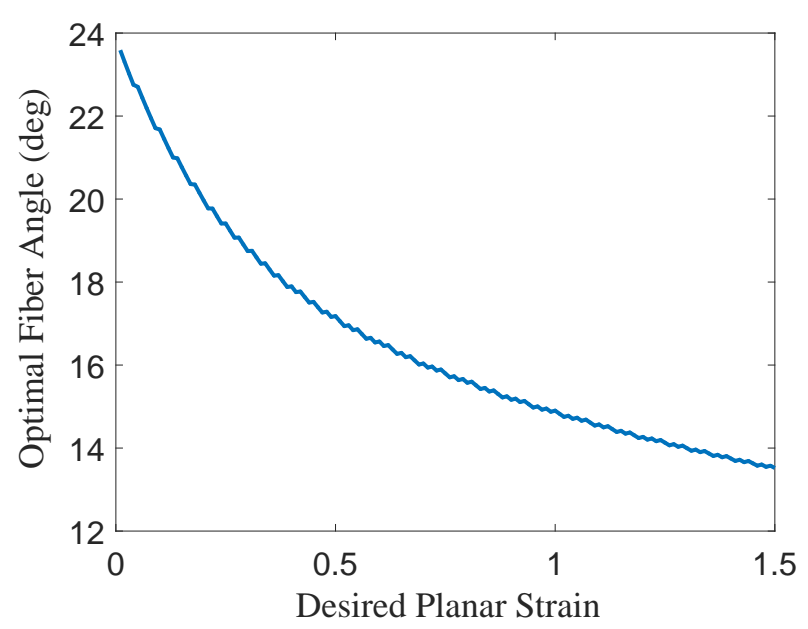

(a)

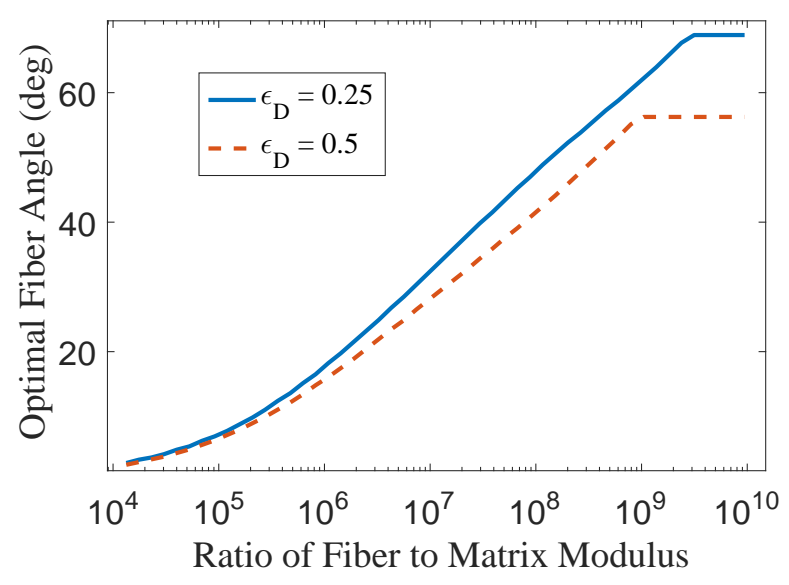

(b)

Figure 13: The fiber angle that minimizes work required to create a given deformation vs. (a) the desired planar strain output, and vs. (b) the ratio of fiber to matrix modulus.

potential energy stored in both the matrix and fiber array for this deformation is shown in figure 12. It can be seen that as the initial fiber angle increases the potential energy stored in the elastomeric matrix decreases due to the fact that a lower compressive strain is required to reach the prescribed planar strain. Conversely, the potential energy stored in the fibers increases exponentially with the increased initial fiber angle, since at higher angles more tension must be placed on the fibers to drive the asymmetric planar expansion. For this case there is an optimal initial fiber angle around $19^{\circ}$ which balances the decrease in required compressive strain with the increase in fiber tension and sheet stiffness.

The optimal fiber orientation is sensitive to several parameters, the most obvious being the planar strain prescribed at the start. We generated soft composite energy storage curves, like the one seen in figure 12, and determined the initial fiber angle that minimized the work required to create a range of planar deformations ranging from $\epsilon_{x x}=0$ to $\epsilon_{x x}=1.5$. The optimal fiber angle is plotted with respect to the desired planar strain in figure 13 a. In general the optimal fiber angle decreases as the required planar strain increases, because at higher strains the large tensions cause the potential energy stored in the fibers to dominate the system potential energy.

The tensile modulus of the reinforcing fibers and the elastic modulus of the matrix also largely affect the interplay of energy storage and optimal reinforcing fiber angle. This interplay can be characterized by the ratio of the two moduli. Though we will not consider soft composites with a very low ratio of fiber to elastomer modulus, since this would violate the inextensible fiber approximation, we calculated optimal fiber angles for a range of modulus ratios for two required strains. Figure $13 \mathrm{~b}$ shows the optimal fiber angle for cases with required planar strains of 0.25 and 0.5 , and a range of modulus ratios. It can be seen that as the modulus ratio increases so does the optimal fiber angle, since the stiffer fibers will experience less deformation and hence less potential energy storage. At the very high modulus ratio values we see that the optimal fiber angle levels off indicating that the fiber stiffness is so high that any extension in the fibers becomes insignificant, along with the energy storage in the fibers, and any further increase in stiffness no longer affects the energy dynamics in the sample. It should also be noted that the change in optimal fiber angle between the two desired planar strains is much less than the change in optimal fiber angle reached by adjusting the 


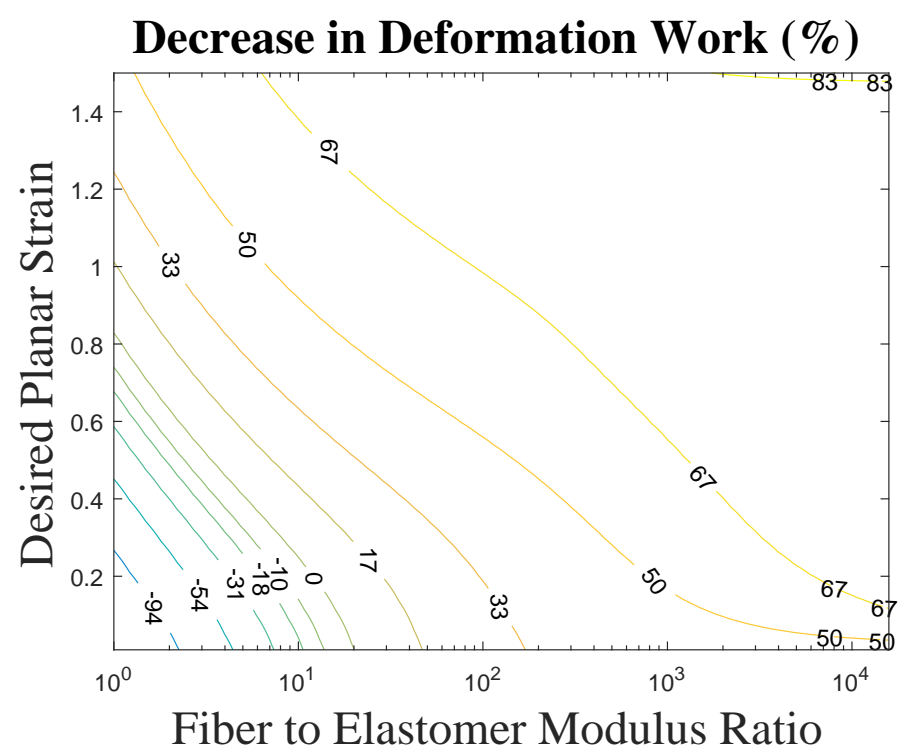

Figure 14: Variation in the optimal fiber angle which minimizes deformation work due to increasing/decreasing the ratio of fiber to matrix modulus.

modulus ratio. This is promising from a design perspective, since we would like to optimize soft composite design with respect to the materials employed as opposed to any specific action or required planar strain.

Here it should be noted that the optimal reinforcement analysis has not yet considered the option of "no reinforcement'. We, therefore, calculated the required work to deform an unreinforced homogeneous elastomer sample over a similar range of desired planar strains and fiber to elastomer modulus ratios, along with the work required to create the same planar deformation at the optimal fiber orientation. Figure 14 shows contours of decrease in required deformation energy, due to inclusion of optimal fiber reinforcement, over the desired strain modulus ratio space. It can be seen that for low desired strains and low modulus ratios, deforming the unreinforced elastomer requires less work, but as the desired strains or modulus ratio become large the fiber reinforced soft composites require significantly less energy because of the decrease in necessary compressive strain. The decrease in required deformation energy reaches as high as a $70 \%$ improvement.

The model assumes that the basic fiber element is small compared to the overall size of the composite sheet, so that the planar strains can be considered uniform throughout the sample. Therefore, the kinematics and strain relationships discussed in section 2 do not depend on the density/spacing of reinforcing fibers. However, as can be seen from equations 14 and 19 the stress in the z-direction and the potential energy stored in the fibers both scale with the inverse of the characteristic length $l_{1}$, meaning that the stress and energy storage dynamics as well as the optimal fiber angle do depend on the fiber density. As the density of reinforcing fibers increases, the characteristic length scale of the basic fiber element, $l_{1}$, decreases resulting in a larger required stress to achieve a given compression and a larger portion of energy being stored by the fibers relative to the elastomer matrix. Since $\sigma_{z z}, U_{\text {matrix }}$, and $U_{\text {fibers }}$ all scale with $l_{1}$ to the same proportionality that they scale with the elastomer modulus $E$, an increase in fiber density can be considered an equivalent action to decreasing the modulus ratio. 


\section{Conclusion} reinforcement in these systems is not only used to increase material stiffness, but to restrict body deformation to a set of possible geometries as well. Such reinforcement in squid mantles has allowed specialized and effective propulsive jetting, but sacrificed the versatility seen in unreinforced octopus mantle structures. This paper investigates the strain properties of fiber reinforcement in highly deformable composite sheets. Specifically, we investigate the strain compressed, as seen in EAP and other soft actuator applications.

The strain dynamics are modeled geometrically, defining a null space of possible deformations satisfying the inextensibility of the reinforcing fibers. The strain modeling was observed to be quite accurate, with only minor errors when the sample compression reached a theoretical maximum causing the minimal stretching in the fibers. It was also observed that the planar strain in a desired direction was increased as much as 16 times the resulting strain of an unreinforced sample for a given driving $z$ strain. The stress-strain relationship in the composite sheet was modeled by treating the elastomeric matrix and reinforcing fibers as separate isotropic systems that interact through local stresses as required to create the modeled strain dynamics.

Finally, by integrating the stress over various sheet compressions, we calculated the total energy required to create different deformations with a variety of composite sheet properties. It is shown that for the materials used to create the soft composite sheets optimal fiber angles lie between $15^{\circ}$ and $25^{\circ}$ to minimize work required for deformation, with optimal fiber angle decreasing with increased desired deformation. The optimal fiber angle increases as high as $68^{\circ}$ if the ratio of the fiber to matrix modulus is increased to the point where little to no potential energy is stored in the fibers.

Future studies will improve the modeling of the volumetric strain, examine non-uniform loading distributions $\left(\sigma_{z z}=f(x, y)\right)$, and extend the functional relationships to non-Cartesian geometries.

\section{Acknowledgments}

This work is supported by a grant from the Office of Naval Research.The Authors would like to sincerely thank Antonio Johman and Zach Smythurst for their assistance in fabricating samples, and compiling node locations from sample images. Thanks for all the time counting pixels guys.

\section{References}

[1] M. Krieg, K. Mohseni, New perspectives on collagen fibers in the squid mantle, Journal of Morphology 273 (6) (2012) 586-595. doi:10.1002/jmor.20003

URL http://dx.doi.org/10.1002/jmor.20003

${ }_{545}[2]$ M. J. Lighthill, Hydrodynamics of aquatic animal locomotion, Annual Review of Fluid Mechanics 1 (1969) 413445. doi:10.1146/annurev.fl.01.010169.002213

URL http://dx.doi.org/10.1146/annurev.fl.01.010169.002213 
[3] W. M. Kier, Biomechanics (Structures and Systems): A Practical Approach, IRL Press at Oxford University, Oxford, England, 1992, Ch. Hydrostatic skeletons and muscular hydrostats, p. 11.

[4] W. M. Kier, K. K. Smith, Tongues, tentacles and trunks: the biomechanics of movement in muscular hydrostats, Zoological Journal of the Linnean Society 83 (1985) 307-324.

[ [5] J. M. Gosline, M. E. DeMont, Jet-propelled swimming in squids, Scientific American 252 (1985) 96-103. doi: 10.1137/0911052.

URL http://dx.doi.org/10.1137/0911052

[6] D. V. Ward, S. A. Wainwright, Locomotory aspects of squid mantle structure, Journal of Zoology 167 (4) (1972) 437-449. doi:10.1111/j.1469-7998.1972.tb01735.x. URL http://dx.doi.org/10.1111/j.1469-7998.1972.tb01735.x

[7] S. A. Wainwright, W. D. Biggs, J. D. Currey, J. M. Gosline, Mechanical Design in Organisms, Princeton University Press, Princeton, NJ, USA, 1976.

[8] S. Vogel, Life in Moving Fluids, 2nd Edition, Princeton University Press, Princeton, NJ, 1994.

[9] Q. Bone, A. Pulsford, A. D. Chubb, Squid mantle muscle, Journal of the Marine Biological Association of the United Kingdom 61 (2) (1981) 327-342. doi:10.1017/S0025315400046981. URL http://dx.doi.org/10.1017/S0025315400046981

[10] J. M. Gosline, R. E. Shadwick, The role of elastic energy-storage mechanisms in swimming: An analysis of mantle elasticity in escape jetting in the squid Loligo-Opalescens, Canadian Journal of Zoology 61 (6) (1983) 1421-1431. doi:10.1139/z83-191.

URL http://dx.doi.org/10.1139/z83-191

[11] J. M. Gosline, J. D. Steeves, A. D. Harman, M. E. Demont, Patterns of circular and radial mantle muscle-activity in respiration and jetting of the squid Loligo-opalescens, Journal of Experimental Biology 104 (1983) 97-109.

[12] P. Macgillivray, E. J. Anderson, G. M. Wright, M. E. DeMont, Structure and mechanics of the squid mantle, Journal of Experimental Biology 202 (1999) 683-695.

[13] J. Thompson, W. Kier, Ontogenetic changes in fibrous connective tissue organization in the oval squid, sepioteuthis lessoniana lesson, 1830, Biology Bulletin 201 (2001) 136-153.

[14] R. K. O’Dor, D. M. Webber, Invertebrate athletes: Trade-offs between transport efficiency and power density in cephalopod evolution, Journal of Experimental Biology 160 (1991) 93-112.

[15] E. J. Anderson, M. A. Grosenbaugh, Jet flow in steadily swimming adult squid, Journal of Experimental Biology 208 (2005) 1125-1146. doi:10.1242/jeb.01507.

URL http://dx.doi.org/10.1242/jeb.01507

[16] I. A. Anderson, T. A. Gisby, T. G. McKay, B. M. O’Brien, Multi-functional dielectric elastomer artifical muscles for soft and smart machines, J. Applied Phys. 112 (2012) 041101-1:041101-19. 
[17] R. Perline, R. Kornbluh, Q. Pei, J. Joseph, High-speed electrically actuated elastomers with strain greater than $100 \%$, Science 5 (287) (2000) 836-839.

[18] J. Krey, K. Friedrich, Variably flexible aramid fibre composites with elastomeric matrices, Plastics and Rubber Processing and Applications 11 (2).

[19] B. D. Agarwal, L. J. Broutman, Analysis and performance of fiber composites, John Wiley \& Sons, New York, 1980.

[20] S. K. Clark, Composite theory applied to elastomers, Tech. Rep. CR-180322, N87-27035, NASA (1987).

[21] S. Y. Lou, T. W. Chou, Finite deformation of flexible composites, Proc. Roy. Soc. Lon. A 429 (1877) (1990) $569-586$.

[22] L. D. Peel, Fabrication and mechanics of fiber-reinforced elastomers, Ph.D. thesis, Bringham Young University, Utah (1998).

[23] Z. Zhang, M. Philen, Pressurized artifical muscles, J. Intell. Mater. Syst. Struct. 23 (3) (2012) 255-268.

[24] R. Lakes, K. Wojciechowski, Negative compressibility, negative Poisson's ratio, and stability, Physica Status Solidi 245 (3) (2008) 545-551.

[25] J. M. Gere, S. P. Timoshenko, Mechanics of Materials, 4th Edition, Brooks/Cole, Boston, MA, USA, 1997. 